\title{
Red Cell Alloantibody Screening: Comparative Analysis of Three Different Technologies
}

\author{
Nicoletta Orlando Maria Bianchi Caterina Giovanna Valentini Maddalena Maresca \\ Giuseppina Massini Rossana Putzulu Gina Zini Luciana Teofili
}

Istituto di Ematologia, Università Cattolica del Sacro Cuore - Fondazione Policlinico Universitario A. Gemelli, Rome, Italy

\section{Keywords}

Red blood cell alloantibodies - Type \& Screen . Diagnostic accuracy · Individualized medicine .

Predictive assays

\section{Summary}

Background: The detection of irregular antibody is a critical issue in the management of red blood cell transfusion according to the Type \& Screen (T\&S) practice. In order to implement the T\&S procedure at our blood bank, we compared three different automated analyzers based on column agglutination technique (CAT) or solid phase red cell adherence assay (SPACA) methods. Methods: Pre-transfusion antibody screening was performed in 986 patients candidate to elective surgery at low risk for red blood cell transfusion. We tested the following kits: the three-cell panel micro-CAT system ID-DiaCell I-IIIII (DiaMed), the four-cell panel solid-phase system Capture-R Ready Screen-4 (Immucor), and the four-cell panel micro-CAT system Serascan Diana-4 (Grifols). Positive results were further investigated using corresponding identification panels, and discrepant results were investigated with all the antibody identification systems. Results: Among 986 samples, we observed 967 concordant negative results $(98.1 \%), 8$ concordant positive results $(0.8 \%$ of cases), and 11 discrepant results (1.1\%). Among discrepant samples, an alloantibody could been identified in two patents (anti-M, detected by Serascan Diana-4 and ID-DiaCell I, II, III; anti-Kp ${ }^{\text {, }}$, detected by Capture-R Ready Screen-4 and Serascan Diana-4). Conclusion: Among the evaluated technologies, the four-cell panel micro-CAT system displayed the highest sensitivity and specificity with an optimal negative predictive value. These features might be relevant to the routine implementation of the T\&S transfusion strategy.

(C) 2018 S. Karger GmbH, Freiburg

\section{Introduction}

Pre-transfusion testing is necessary to assure $\mathrm{ABO}$ and $\mathrm{Rh}$ type compatibility between donor and patient blood, as well as to disclose the presence of red blood cell (RBC) alloantibodies potentially causing transfusion reaction. The cross-match (XM; i.e., testing the patient's serum against the donor's RBCs) is intended as the check for $\mathrm{ABO}$ compatibility and for the detection of alloantibodies. It represents the most important pre-transfusion testing used in routine practice.

Since many surgical procedures rarely require blood transfusion, there is no need to reserve blood units in the blood bank for each specific patient, provided that the laboratory can quickly deliver components in case of emergency. The Type and Screen (T\&S) is a reliable alternative for the management of these situations $[1,2]$. The T\&S includes typing the patient's RBCs for ABO and $\mathrm{Rh}$ blood groups, and screening patient's serum for the presence of alloantibodies. If the antibody screen is negative, it can be predicted that more than $99.99 \%$ of RBC units electronically matched for $\mathrm{ABO}$ groups will be compatible in the XM test [3]. Since 1984, the American Association of Blood Banks (AABB) standards has stated that the XM can be omitted if the antibody screening is nonreactive [4]. Nowadays, procedures for blood product release according to the T\&S and electronic XM have been adopted in several European countries, and their implementation has been advocated by the Italian transfusion regulation as well [5]. Actually, the adoption of T\&S procedure and electronic XM in selected patient populations support the rational allocation of blood resources $[1,2,4]$.

The method for screening irregular antibodies is pivotal to select patients candidate to the T\&S procedure. Actually, the ideal screening should allow the detection of all clinically relevant antibodies without capturing clinically insignificant antibodies or nonspecific reactions, with acceptable sensitivity and specificity. Basically, RBC antibody screening and identification are carried out by agglutination or solid-phase red cell adherence assay (SPRCA)

\section{KARGER}

() 2018 S. Karger GmbH, Freiburg

Fax +497614520714 
Table 1. Antibody screening and antibody identification results

\begin{tabular}{llll}
\hline \multirow{2}{*}{ Technique } & System & \multicolumn{2}{l}{ Antibody screening } \\
\cline { 3 - 4 } & & negative & positive \\
\hline Micro-CAT & ID-DiaCell I-II-III (Diamed) & $975(98.8 \%)$ & $11(1.1 \%)$ \\
Micro-CAT & Serascan Diana-4 (Grifols) & $974(98.7 \%)$ & $12(1.2 \%)$ \\
SPRCA & Capture-R Ready-Screen (Immucor) & $970(98.4 \%)$ & $16(1.6 \%)$ \\
\hline \multicolumn{2}{l}{ Micro-CAT } & M Micro-column agglutination technique; SPRCA = solid-phase red cell agglutination assay.
\end{tabular}

Micro-CAT = Micro-column agglutination technique; SPRCA = solid-phase red cell agglutination assay. methods. Although conventional test tube technique has been long considered the reference method, nowadays column agglutination technique (CAT) and SPRCA are widely employed [6, 7]. Generally, both methods have shown similar or even higher sensitivity and specificity when compared with classical tube testing $[8,9]$. Importantly, they can be run in automation, require small volumes of both samples and reagents, avoid subjective interpretation, and produce reliable results [8].

Our hospital is a tertiary academic hospital and about 10,400 tests for antibody screening are approximately performed per year. The antibody screening is usually accomplished in automation by micro-column agglutination with the IH-1000 System (Biorad Laboratories, Hercules, CA, USA), which uses a three-cell panel (ID-DiaCell I-II-III; DiaMed GmbH, Cressier, Switzerland). Positive samples are further investigated with the 11-cell panel system papainized and not papainized (ID-DiaPanel and ID-DiaPanel P; DiaMed). In view of implementing the T\&S procedure, we undertook this study in order to evaluate additional technologies for alloantibody detection. Therefore, we prospectively investigated alloantibodies in patients, whose blood product requirements were potentially amenable by the T\&S procedure, using three different automated analyzers based on CAT or SPACA methods. The results obtained were then compared in terms of sensitivity and specificity.

\section{Material and Methods}

\section{Study Population}

From April 2015 to November 2016, patients candidate to transfusion therapy for elective surgery were analyzed. Patients were selected on the basis of a low risk for transfusions, considering the diagnosis, kind of intervention, and clinical conditions. According to the scheduled provision of reagents, a median number of 48 consecutive patients per month could be investigated with the three antibody screening systems. The inclusion of repeated patients was omitted. Detailed clinical information necessary to quantify the transfusion risk for each patient were available in electronic databases in use at our hospital. The study was approved by the local Institutional Review Board.

\section{Antibody Screening}

The automated analyzers based on micro-column agglutination were the IH-1000 System (Biorad Laboratories), which uses a three-cell panel (ID-DiaCell I-II-III; DiaMed) and the Erytra (Grifols, Barcelona, Spain) which uses a four-cell panel (Serascan Diana-4; Grifols). The automated analyzer based on SPRCA was the Immucor Neo (Immucor Inc., Norcross, GA, USA), which utilizes a four-cell panel (Capture-R, Ready-Screen; Immucor). Whole blood samples (EDTA, $6 \mathrm{ml}$ ) were tested within $24 \mathrm{~h}$ from collection. All tests were performed according to manufacturers' instructions. Automated reading of reactions was obtained through the artificial vision technology employed on each platform. Results of screening tests were given on a scale of strength from nega- tive to positive (from $1+$ to $4+$ ). In case of plus/minus or weak reactions, the screening was repeated; if results were confirmed, samples were considered positive and then subjected to the antibody identification. A qualified operator reviewed all unclear results.

\section{Antibody Identification}

In samples with positive screening, the alloantibody specificity was investigated using the corresponding apposite panels. Samples positive at solid-phase screening (Immucor) were investigated with a 13-cell panel (Capture-R ReadyID; Immucor). Samples positive at micro-column screening (Biorad and Grifols) were investigated with a 11-cell panel (ID-DiaPanel; DiaMed) or with a 15-cell panel (Identisera Diana/Extend; Grifols). Moreover, samples identified as positive at micro-column screening were further analyzed by the corresponding enzyme-treated cell panels (ID-DiaPanel P Identisera DianaP/Extend P). All samples with discrepant results among the different systems were investigated with all antibody identification systems mentioned above. All tests were performed according to manufacturers' instructions.

\section{Analysis of Results}

Results were compared to identify discrepancies among methods. Results were considered discrepant when the sample turned out positive in one method and negative in the other one or two methods. In case of discrepancy, antibody identification was carried out as mentioned above, in order to confirm the antibody screening results. Samples were considered true-positive when the positive test in the antibody screening was associated with the identification of the antibody specificity in at least one of the systems utilized for the antibody identification. On the opposite, samples were considered false-positive when the positive results in the antibody screening were not followed by subsequent specificity antibody identification. We calculated accuracy, sensitivity, specificity, positive predictive value (PPV), and negative predictive value (NPV) of each method by standard formulae, including the $95 \%$ confidence intervals (CI) [10]. The test sensitivity was estimated as the proportion of subjects with the target condition in whom the test was positive. Similarly, the test specificity was estimated as the proportion of subjects without the target condition in whom the test was negative.

\section{Results}

\section{Antibody Screening and Identification}

Overall, 986 patients (382 males and 604 females) were evaluated; the median age was 63 years (range 3-98 years). The results of $\mathrm{RBC}$ antibody screening obtained in the three different systems (ID-DiaCell I-II-III, Serascan Diana-4, and Capture-R ReadyScreen), are illustrated in table 1 . Overall, we observed concordant results in 975 patients $(98.8 \%)$ - 967 concordant negative (98.1\%) and 8 concordant positive $(0.8 \%$ of cases $)$ results were obtained (table 1). In addition, 11 samples (1.1\% of cases) produced discrepant results among the three evaluation systems (table 2 ). The reaction strength observed in discrepant cases ranged from \pm to $2+$ (table 2). 
Table 2. Discrepant results occurred among 986 evaluated patients. Antibody screening, strength reaction and antibody identification are shown

\begin{tabular}{|c|c|c|c|c|}
\hline \multirow[t]{3}{*}{ Patients } & \multicolumn{3}{|c|}{ Antibody screening } & \multirow[t]{3}{*}{ Antibody identification } \\
\hline & ID-DiaCell & Serascan & Capture-R & \\
\hline & I-II-III & Diana-4 & Ready-Screen-4 & \\
\hline 1 & positive $(2+)$ & positive $(2+)$ & negative & unspecified reaction \\
\hline 2 & positive $(1+)$ & positive $(1+)$ & negative & unspecified reaction \\
\hline 3 & positive $(1+)$ & positive $(2+)$ & negative & anti-m \\
\hline 4 & negative & negative & positive $(1+)$ & unspecified reaction \\
\hline 5 & negative & negative & positive $(1+)$ & unspecified reaction \\
\hline 6 & negative & negative & positive $(1+)$ & unspecified reaction \\
\hline 7 & negative & negative & positive $(1+)$ & unspecified reaction \\
\hline 8 & negative & negative & positive $(1+)$ & unspecified reaction \\
\hline 9 & negative & negative & positive (1+) & unspecified reaction \\
\hline 10 & negative & negative & positive $( \pm)$ & unspecified reaction \\
\hline 11 & negative & positive $(2+)$ & positive $(1+)$ & anti-Kp ${ }^{\mathrm{a}}$ \\
\hline
\end{tabular}

\begin{tabular}{ccrlll}
\hline Patients & Sex & Age & Diagnosis and elective surgery & $\begin{array}{l}\text { Previous } \\
\text { transfusions }\end{array}$ & $\begin{array}{l}\text { Previous } \\
\text { pregnancies }\end{array}$ \\
\hline 1 & M & 78 & neurosurgery & no & - \\
2 & F & 3 & abdominal trauma & no & - \\
3 & F & 74 & gastric cancer & no & no \\
4 & M & 80 & colon cancer & no & - \\
5 & F & 46 & cesarean section in twin birth & no & yes \\
6 & M & 74 & rectus cancer & no & - \\
7 & F & 33 & uterine leiomyoma & no & no \\
8 & F & 73 & ovarian cancer & no & yes \\
9 & M & 66 & TURP & no & - \\
10 & F & 79 & knee arthroprosthesis & no & yes \\
11 & M & 58 & CABG & no & - \\
\multicolumn{7}{l}{ TUR $=$ Transurethral retrograde prostatectomy; CABG = coronary artery bypass graft surgery. } \\
\hline
\end{tabular}

Table 3. Clinical data of patients displaying discrepant results
Table 4. Result distribution among different systems

\begin{tabular}{lccc} 
& $\begin{array}{l}\text { ID-DiaCell } \\
\text { I-II-III }\end{array}$ & $\begin{array}{l}\text { Serascan } \\
\text { Diana-4 }\end{array}$ & $\begin{array}{l}\text { Capture-R } \\
\text { Ready-Screen }\end{array}$ \\
\hline True-positive & 9 & 10 & 9 \\
False-positive & 2 & 2 & 7 \\
True-negative & 974 & 974 & 969 \\
False-negative & 1 & 0 & 1 \\
\hline
\end{tabular}

\section{Analysis of Results}

Overall, the ID-DiaCell I-II-III system produced 975 negative results and 11 positive results (table 1 ), with one false-negative result due to the missed identification of Anti-Kp ${ }^{\mathrm{a}}$ alloantibody, and 974 true-negative results. On the opposite, among the 11 positive reactions, 9 results were true-positive and 2 results were false-positive (table 4). The antibody screening in Serascan Diana-4 led to 974 negative results and 12 positive results (table 1). No false-negative reactions occurred in the Serascan Diana-4 system; moreover, among the 12 positive reactions, 10 were true-positive and 2 were false-positive results (table 4). The Capture-R Ready-Screen-4 system produced 970 negative results and 16 positive results (table 1 ). One out of 970 negative results was false-negative due to the missed identification of an anti-M alloantibody, while 969 samples 
Table 5. Diagnostic accuracy, sensitivity and specificity of ID-DiaCell I-II-III, Serascan Diana4, and Capture-R Ready-Screen systems

\begin{tabular}{lccc}
\hline & $\begin{array}{l}\text { ID-DiaCell I-II-III } \\
\%(95 \% \mathrm{CI})\end{array}$ & $\begin{array}{l}\text { Serascan Diana-4 } \\
\%(95 \% \mathrm{CI})\end{array}$ & $\begin{array}{l}\text { Capture-R Ready-Screen } \\
\%(95 \% \mathrm{CI})\end{array}$ \\
\hline $\begin{array}{lll}\text { Accuracy } \\
\text { Sensitivity }\end{array}$ & $99.7(0.990-0.999)$ & $99.8(0.992-1.000)$ & $99.2(0.983-0.996)$ \\
Specificity & $99.8(0.992-1.000)$ & $100(0.995-1.000)$ & $90.0(0.879-0.918)$ \\
PPV & $81.8(0.792-0.841)$ & $99.8(0.992-1.000)$ & $99.3(0.985-0.997)$ \\
NPV & $99.9(0.993-1.000)$ & $83.3(0.808-0.856)$ & $56.3(0.531-0.594)$ \\
\hline Accuracy = (true-positives + true-negatives) / (true-positives + false-positives + true-negatives + false- \\
negatives); sensitivity = true-positives / (true-positives + false-positives); specificity = true-negatives / \\
(true-negatives + false-positives); PPV = true-positives / (true-positives + false-positives); NPV = true- \\
negatives / (true-negatives + false-negatives).
\end{tabular}

were true-negative. Nine out of 16 positive samples were true-positive, while 7 were false-positive (table 4). The accuracy, sensitivity, specificity, PPV, and NPV were calculated for all systems and are illustrated in table 5 .

\section{Discussion}

In this study we compared the capacity of different technologies based on micro-column agglutination (ID-DiaCell I-II-III and Serascan Diana-4) or solid-phase agglutination (Capture-R ReadyScreen) to detect RBC alloantibodies in a routine screening scenario. Since this study was undertaken in order to implement the T\&S procedure at our blood bank, the primary goal of this comparative analysis was to identify which methodology had the maximal sensitivity, in order to avoid transfusing incompatible RBCs to patients with undetected alloantibodies. Therefore, a series of patients with negative transfusion history, and ideally candidate to receive $\mathrm{RBC}$ products according to the $\mathrm{T} \& \mathrm{~S}$ and electronic $\mathrm{XM}$ pre-transfusion procedure, were screened for RBC alloantibodies in parallel with all the above mentioned systems.

As expected, concordant results were obtained in the vast majority of cases (975 out of $986,98.9 \%$ ), including 8 patients with positive reactions. The prevalence of patients with irregular antibodies in the entire cohort was extremely low, representing about $1 \%$ of the entire series. Although the number of positive results was not impressive, the majority of these clinically significant alloantibodies, such as anti-D, anti-Cw, anti-Kell and anti-Jka, were detected by all methodologies employed, confirming their high accuracy level. Nevertheless, in two cases alloantibodies were not detected by the ID-DiaCell I-II-III (anti-Kp ${ }^{\mathrm{a}}$ ) or by the Capture-R Ready-Screen (anti-M). The anti-M is a fairly common naturally occurring antibody; in general, it has no clinical impact in transfusion practice. It is predominantly of IgM type, with optimal thermal reactivity at $4{ }^{\circ} \mathrm{C}$ and very rarely causes delayed hemolytic transfusion reaction (HTR) or hemolytic disease of the fetus and newborn (HDFN) [1113]. Nonetheless, few cases of anti-M antibodies reactive at room temperature and at $37^{\circ} \mathrm{C}$ have been reported in patients of different ages and diagnosis [14]. The anti-M was not detected by the Capture-R Ready-Screen system because IgG-coated indicator RBCs used in this system can bind only to IgG alloantibodies. On the op- posite, both micro-column affinity gel systems are able to detect IgM alloantibodies as they contain polyspecific anti-human globulins, which bind either directly (Serascan Diana-4) or indirectly through the light chain (ID-DiaCell I-II-III) to the IgM molecules. The anti-Kpa antibody was captured only by the four-cell panel system (Serascan Diana-4 and Capture-R Ready-Screen), whilst it was overlooked by the three-cell panel system. The anti-Kp $\mathrm{p}^{\mathrm{a}}$ is directed against a low-frequency antigen of the Kell group [15]. Actually, about $98 \%$ of Caucasian donors and $100 \%$ of Black donors are $\mathrm{Kp}^{\mathrm{a}}$ negative [15]. Therefore, most commercially available screening kits do not contain $\mathrm{Kp}^{\mathrm{a}}$-positive cells. Nonetheless, it is widely acknowledged that anti-Kp $\mathrm{a}^{\mathrm{a}}$ antibody may cause severe HTR and HDFN [16-22]. Indeed, despite the infrequent occurrence, its investigation through $\mathrm{Kp}^{\mathrm{a}}$-positive screening cell panels is undoubtedly worthy in clinical practice.

As previously reported, also in this study the SPRCA methodology detected more nonspecific reactivity and panagglutinins than CAT did [23]. In particular, the Immucor system found 7 positive results that at the subsequent work-up did not result in any antibody identification (table 2). Recently, solid-phase unidentified reactions have been associated with female sex or diagnoses like cancer, pregnancy, surgery, and trauma, and data gathered in our patients are in line with these observations [24]. Although it has been recently reported that a minority of patients with nonspecific reactivity at SPRCA might develop new auto- or alloantibodies, the majority of them tend to become definitely negative at following alloantibody screenings, thus confirming the elusive nature of these results $[24,25]$. In our scenario, however, consisting in patients at very low transfusion risk, the false-positive results could require additional investigations finally resulting in the delay of surgical procedures.

Overall, our study exhibit an important limitation due to the low number of positive samples in the investigated population. In particular, there are several alloantibodies in addition to those we detected that can be relevant in the transfusion therapy management of immunized patients. All investigated automated systems in the present study showed high sensitivity and specificity (ranging between 90 and $100 \%$ or 99.3 and $99.8 \%$ ). Nevertheless, the low prevalence of alloantibodies in our series of patients resulted in a variable rate of positive predictive values, ranging from $56.3 \%$ for Capture-R Ready-Screen to $81.8 \%$ for ID-DiaCell I-II-III and 
83.3\% for Serascan Diana-4. Our observations confirm the low specificity of the solid-phase assay system and suggest that the utilization of $\mathrm{Kp}^{\mathrm{a}}$-containing four-cell panels increases the screening sensitivity. These findings may be relevant to the implementation of the T\&S transfusion strategy in a patient population with a very low prevalence of alloimmunization. Future studies detecting additional clinical relevant alloantibodies are advisable to complement our results.

\section{Disclosure Statement}

N.O. received a study grant from Immucor for the research described in this paper. The authors declare no further conflicts of interest.

\section{References}

1 Boral LI, Henry JB: The type and screen: a safe alternative and supplement in selected surgical procedures. Transfusion 1977;17:163-168.

2 Alavi-Moghaddam M, Bardeh M, Alimohammadi H, Emami H, Hosseini-Zijoud SM: Blood transfusion practice before and after implementation of type and screen protocol in emergency department of a university affiliated hospital in Iran. Emerg Med Int 2014; 2014:316463.

3 Powers A, Chandrashekar S, Mohammed M, Uhl L Identification and evaluation of false-negative antibody screens. Transfusion 2010;50:617-621.

4 Standards for Blood banks and Transfusion Services, 30th ed. Bethesda, American Association of Blood Banks, 2016.

5 Ministero della Salute: Decreto 2 novembre 2015. Disposizioni relative ai requisiti di qualità e sicurezza del sangue e degli emocomponenti. www.avis.it/wp-content/uploads/2016/08/GU-SG-n_300-del-28-12-2015_ SO_069.pdf (last accessed February 21, 2018).

6 Voak D: Validation of new technology for antibody detection by antiglobulin tests. Transfus Med 1992;2: 177-179.

7 Bajpai M, Kaur R, Gupta E: Automation in immunohematology. Asian J Transfus Sci 2012;6:140-144.

8 Garg S, Saini N, Bedi KR, Basu S: Comparison of micro column technology with conventional tube methods for antibody detection. J Lab Physicians 2017;9:95-99.

9 Tenali EL, Watson CR: The evaluation of solid phase and gel technology in antibody screening and identification: a comparative study (P1488). Proceedings of the ISBT Congress, Oslo, June 2 to July 2, 1998.
10 US Food and Drug Administration: Guidance for Industry and FDA Staff. Statistical Guidance on Reporting Results from Studies Evaluating Diagnostic Tests. www.fda.gov/RegulatoryInformation/Guidances/ ucm071148.htm (last accessed February 21, 2018).

11 Alperin JB, Riglin H, Branch DR, Gallagher MT, Petz LD: Anti-M causing delayed hemolytic transfusion reaction. Transfusion 1983;23:322-324.

12 Sancho JM, Pujol M, Fernández F, Soler M, Manzano P, Feliu E: Delayed hemolytic transfusion reaction due to anti-M antibody. Br J Haematol 1998;103:268-269.

13 Tondon R, Kataria R, Chaudhry R: Anti-M: report of two cases and review of literature. Asian J Transfus Sci 2008;2:81-83.

14 Shah SP, Kalgutkar SM, Sawant RB, Deshpande AS. Anti-M antibodies: biphasic (reactive at room temperature and at $37^{\circ} \mathrm{C}$ ): a case series. Asian J Transfus Sci 2016;10:159-160.

15 Dean L: Chapter 8 The Kell blood group; in Blood Groups and Red Cell Antigens Bethesda, National Center for Biotechnology Information (US), 2005. www.ncbi.nlm.nih.gov/books/NBK2270/ (last accessed February 21, 2018).

16 Geczy A, Ennis J, Golian PR: A further example of anti-Kpa. Transfusion 1965;5:89-91.

17 Smoleniec J, Anderson N, Poole G: Hydrops fetalis caused by a blood group antibody usually undetected in routine screening. Arch Dis Child Fetal Neonatal Ed 1994;71:F216-217.

18 Costamagna L, Barbarini M, Viarengo GL, Pagani A, Isernia D, Salvaneschi L: A case of hemolytic disease of the newborn due to anti-Kpa. Immunohematology 1997;13:61-62.
19 Koshy R, Patel B, Harrison JS: Anti-Kpa-induced severe delayed hemolytic transfusion reaction. Immunohematology 2009;25:44-47.

20 Tuson M, Hue-Roye K, Koval K, Imlay S, Desai R, Garg G, Kazem E, Stockman D, Hamilton J, Reid ME: Possible suppression of fetal erythropoiesis by the Kell blood group antibody anti-Kp(a). Immunohematology 2011;27:58-60.

21 Brumbaugh JE, Morgan S, Beck JC, Zantek N, Kearney S, Bendel CM, Roberts KD: Blueberry muffin rash, hyperbilirubinemia, and hypoglycemia: a case of hemolytic disease of the fetus and newborn due to antiKp(a). J Perinatol 2011;31:373-376.

22 Padmore R, Berardi P, Erickson K, Desjardins D, Giulivi A, Tokessy M, Neurath D, Saidenberg E: Acute extravascular hemolytic transfusion reaction due to anti-Kpa antibody missed by electronic crossmatch. Transfus Apher Sci 2014;51:168-171.

23 Yamada C, Serrano-Rahman L, Vasovic LV, Mohandas $\mathrm{K}$, Uehlinger J: Antibody identification using both automated solid-phase red cell adherence assay and a tube polyethylene glycol antiglobulin method. Transfusion;2008;48:1693-1698.

24 Miller NM, Johnson ST, Carpenter E, Naczek CA, Karafin MS: Patient factors associated with unidentified reactivity in solid-phase and polyethylene glycol antibody detection methods. Transfusion 2017;57: 1288-1293.

25 Olofson AM, Chandler RM, Marx-Wood CR, Babcock CA, Dunbar NM: Increased alloimmunisation and transfusion reaction reporting in patients with solidphase panreactivity. J Clin Pathol 2017;70:981-983. 